\title{
Editorial
}

\section{Thin-Film Photovoltaics 2013}

\section{Gaetano Di Marco, ${ }^{1}$ Antonino Bartolotta, ${ }^{1}$ Francesco Bonaccorso, ${ }^{1}$ Stefano Caramori, ${ }^{2}$ Giuseppe Calogero, ${ }^{1}$ and Leonardo Palmisano ${ }^{3}$}

\author{
${ }^{1}$ CNR-IPCF, Istituto per i Processi Chimico-Fisici, Viale Ferdinando Stagno d'Alcontres 37, 98158 Messina, Italy \\ ${ }^{2}$ Dipartimento di Chimica, Università degli Studi di Ferrara, Via L. Borsari 46, 44100 Ferrara, Italy \\ ${ }^{3}$ DEIM, Università degli Studi di Palermo, Viale delle Scienze, Edificio 6, 90128 Palermo, Italy \\ Correspondence should be addressed to Gaetano Di Marco; dimarco@its.me.cnr.it
}

Received 11 December 2013; Accepted 11 December 2013; Published 12 January 2014

Copyright (c) 2014 Gaetano Di Marco et al. This is an open access article distributed under the Creative Commons Attribution License, which permits unrestricted use, distribution, and reproduction in any medium, provided the original work is properly cited.

This special issue is the third edition in a series that has the purpose to collect original contributions by specialists in the field of thin-films photovoltaics (PVs). The fundamental mechanisms at the base of architectures and working principles of PV devices have been investigated both theoretically and experimentally. Particular attention has been paid to key aspects such as electronic transport in semiconducting materials, modelling, structural investigation, sensitizing agents, and so forth, with the aim to increase the efficiency of PV technologies. The contributions are very heterogeneous and although, for some articles, just proofs of principle devices are presented, for others a more viable commercial application can be foreseen. Here we give a brief overview, highlighting the main results, of the articles enclosed in this volume.

In "The effects of malonic acid derivatives and acetic acid derivatives as coadsorbents on the photovoltaic performance of dye-sensitized solar cells", the coadsorption of malonic acid and acid derivatives are instrumental in improving both photocurrent and photovoltage of solar cells. This is probably due to suppression of self-quenching of the excited states and increased electron-injection yields, in parallel to suppression of electron recapture by $\mathrm{I}_{3}^{-}$.

In "Dye-sensitized solar cells with anatase $\mathrm{TiO}_{2}$ nanorods prepared by hydrothermal method", the photoanode in the dye-sensitized solar cell (DSSC) can be optimized by improving the electron collection efficiency, as consequence of the exploitation of nanocrystalline materials with high purity, well-controlled crystallinity, and enhanced surface area such as hydrothermal anatase nanorods with a large surface roughness. These nanostructures can also be associated with $\mathrm{TiO}_{2}$ nanoparticles to prepare highly efficient multiple layer photoanodes.

In "Nanostructured $\mathrm{ZnO}, \mathrm{TiO}_{2}$, and composite $\mathrm{ZnO} / \mathrm{TiO}_{2}$ films for application in dye-sensitized solar cells", efficiency and stability of photoanodes obtained from composite semiconductor films $\left(\mathrm{ZnO} / \mathrm{TiO}_{2}\right)$ and sensitized with the organic molecules Coumarin 343 and Rose Bengal are investigated. It is observed that DSSCs with $\mathrm{ZnO} / \mathrm{TiO}_{2}$ ratio equal to $90 / 10$ have higher stability than the other cells. The performances of DSSCs using various multicomponent electrolytes are assessed.

In "Spectroscopic and morphological studies of metalorganic and metal-free dyes onto titania films for dye-sensitized solar cells", the spectroscopicbehavior of cis-di(thiocyanato) bis(2,2' -bipyridyl-4,4' -dicarboxylato)ruthenium(II) (N719), 3-(5-(4-(diphenylamino)styryl)thiophen-2-yl)-2-cyanoacrylic acid (D5), and a push-pull zinc phthalocyanine sensitizer $(\mathrm{ZnPc})$ adsorbed onto three types of titania thin films with different morphology were investigated. The mesoporous titania films deposited by spin-coating and by doctor-blade were compared with P25 (Degussa) titania deposited by doctor-blade. The use of mesoporous structure allows increasing the dye loading and it is considered promising to realize thin titania photoanodes with high harvesting properties.

In "Effects of hole-collecting buffer layers and electrodes on the performance of flexible plastic organic photovoltaics", the influences on the performance of flexible plastic organic photovoltaic (OPV) devices caused by the sheet resistance of hole-collecting electrodes (ITO) and by the conductivity of a hole-collecting buffer layers are investigated in detail. These 
results indicated that the series resistance originating from the limited surface conductivity may quickly become a major issue to consider in the fabrication of large area cells.

In "Photovoltaic performance of $\mathrm{ZnO}$ nanorod and $\mathrm{ZnO} \mathrm{CdO}$ nanocomposite layers in dye-sensitized solar cells (DSSCs)", a triphenylene diamine sensitizer was synthesized and its absorption spectra, electrochemical behavior, and photovoltaic performance for a potential application in DSSCs were investigated. In the study two different electrodes are employed: $\mathrm{ZnO}$ nanorod and $\mathrm{ZnO}: \mathrm{CdO}$ nanocomposite. The best photovoltaic performance was demonstrated with the $\mathrm{ZnO}: \mathrm{CdO}$ nanocomposite electrode based DSSCs with respect to the $\mathrm{ZnO}$ nanorod electrode one.

In "Effects of anodic buffer layer in top-illuminated organic solar cell with silver electrodes", a relatively efficient molecular planar heterojunction is achieved by spinning a buffer layer of PEDOT:PSS on $\mathrm{Ag}-\mathrm{AgO}_{x}$ anodes. The PEDOT:PSS thin film separates the active layer far from the $\mathrm{Ag}$ anode preventing metal quenching and redistributing the strong internal optical field toward dissociated interface. The thickness and morphology of this anodic buffer layer are the key factors in determining device performances.

In "Effect of bandgap graded absorber on the performance of a-Si $i_{1-x} G e_{x}: H$ single-junction cells with $\mu c-S i O_{x}: H ~ N-t y p e$ layer", the effect of bandgap grading of absorbers on the performance of a- $\mathrm{Si}_{1-x} \mathrm{Ge}_{x}: \mathrm{H}$ cells using $\mu \mathrm{c}-\mathrm{SiO}_{x}: \mathrm{H}$ n-layer is reported. The influence of the $\mathrm{p} / \mathrm{i}$ and the $\mathrm{i} / \mathrm{n}$ grading widths on carrier collection and photon absorption in the cells were evaluated in terms of external quantum efficiency (EQE), total reflection spectra of cells, and the $J-V$ measurement. Compared to those without grading, these cells show improved efficiency from 5.5 to $7.5 \%$.

In "Numerical simulation of luminescent downshifting in top cell of monolithic tandem solar cells", the performances in monolithic tandem solar cells is limited by the short-circuit current density matching between the top and the bottom cells. A theoretical survey of the luminescence downshifting approach for this device, in order to increase the short circuit current density in the top cell, is presented. The PV glass encapsulation material is replaced with Poly(methyl methacrylate) which is doped with diverse kinds of organic dyes.

In "Effectively improved $\mathrm{SiO}_{2}-\mathrm{TiO}_{2}$ composite films applied in commercial multicrystalline silicon solar cells", a sol-gel spin-coating technique for fabricating composite films, alternative to plasma enhanced chemical vapor deposition (PECVD) for fabricating antireflective coatings (ARCs), useful to increase the efficiency of multicrystalline solar cells, is proposed. This result proves that sol-gel-deposited ARCs process has potential applications in manufacturing low-cost, large-area solar cells.

In "Effect of hydrogen content in intrinsic a-Si:H on performances of heterojunction solar cells", the influences of hydrogen content in intrinsic hydrogenated amorphous silicon (i-a-Si:H) on performances of heterojunction (HJ) solar cells are investigated. Simulation and experimental results are performed to evaluate solar cells with higher i-layer hydrogen content and $\mathrm{HJ}$ solar cells with low hydrogen, respectively. The presence of voids and $\mathrm{Si}$ particles is explained from plasma point of view using optical emission spectroscopy.
In "Characterization and modeling of CdS/CdTe heterojunction thin-film solar cell for high efficiency performance", device simulation is used to investigate the photoelectric efficiency in CdTe/CdSc solar cell. The roles of several limiting factors such as back contact Schottky barrier, doping density, thickness, recombination velocity, and extended CdTe layer are examined. Results obtained indicate that higher performance efficiency may be achieved by adding and optimizing an extended CdTe electron reflector layer at the back Schottky contact.

In "Enhanced photoelectrochemical response from copper antimony zinc sulfide thin films on transparent conducting electrode", it is reported that the presence of zinc in copper antimony sulfide thin films influenced the photoelectrochemical performance and in particular gave rise to an enhancement of the photocurrent. A detailed investigation was carried out by changing the stoichiometry of films and the corresponding surface and optical characterization results have been evaluated.

In "Material properties of laser-welded thin silicon foils", the properties of extended monocrystalline silicon base foils consisting of individual thin silicon wafers welded together are studied. The different approaches of (i) laser spot welding with low constant feed speed, (ii) laser line welding, and (iii) keyhole welding were compared. The cross-sections of the foils were analyzed by electron backscatter diffraction to reveal changes in the crystal structure after laser irradiation. The induced internal stress was investigated by micro-Raman analysis. The keyhole welding was found to be the most favorable process to produce thin silicon foils.

In "Advantages of N-type hydrogenated microcrystalline silicon oxide films for micromorph silicon solar cells", a detailed investigation on the advantages to use $\mathrm{N}$-type hydrogenated microcrystalline silicon oxide films in micromorph silicon solar cells is reported. The electrical and optical properties of the micromorph silicon solar cells were evaluated via the open circuit voltage, fill factor and the short circuit density.

In "Enhanced efficiency of GaAs single-junction solar cells with inverted-cone-shaped nanoholes fabricated using anodic aluminum oxide masks", the growth and fabrication of GaAs solar cells with various methods are investigated. Anodized aluminum oxide masks were prepared from an aluminum foil by a two-step anodization method. Nanoholes were formed on the top surface of the solar cells after the anodized aluminum oxide mask removal. Photovoltaic and optical characteristics of the GaAs solar cells in the presence and in the absence of the nanohole arrays and the dependence of the efficiency enhancement on the etching depth of the nanoholes were investigated.

In "Thin film CIGS solar cells, photovoltaic modules, and the problems of modeling", are focused on the methodological problems of modeling at cell structure and photovoltaic module levels for thin film CIGS solar cells. The structural cell simulation and the five-parameter analysis of a commercial CIGS module have been described. From their comparison, the authors have extracted the values of the local parasitic network resistance to be associated with the structural simulation of the cell, thus obtaining a good agreement between the two models. 
In "Reliability analysis of III-V solar cells grown on recycled GaAs substrates and an electroplated nickel substrate", the reliability of III-V solar cell grown on new/recycled GaAs substrate and thin-film solar cell transferred on electroplated $\mathrm{Ni}$ substrate has been explored. The work aims to achieve two basic goals. The first is to provide a thin-film solar cell characterized by high heat dissipation values and, in this context, the thermal conductivity $\kappa$ of $\mathrm{Ni}(=91 \mathrm{~W} / \mathrm{m} \cdot \mathrm{K})$ is better than that of GaAs $(55 \mathrm{~W} / \mathrm{m} \cdot \mathrm{K})$. The second target is to reuse the GaAs substrate after the epitaxial lift-off processing for cost reduction.

In "Optical and structural investigation of CdSe quantum dots dispersed in PVA matrix and its photovoltaic application", the effect of the concentrations of the cadmium source on the optical properties of CdSe quantum dots (QDs) dispersed in polyvinyl alcohol (PVA) matrix thin films was investigated through UV-Vis absorption spectroscopy. The CdSe QDs were synthesized via simple heat induced thermolysis technique. The structural analysis, particle size determination, and morphological studies of the CdSe/PVA nanocomposite thin films were done with the help of X-ray diffraction (XRD) and transmission electron microscopy (TEM). Furthermore a $\mathrm{CdSe} / \mathrm{CdTe}$ based solar cell was prepared and its photovoltaic parameters were measured and reported.

In "Near infrared lateral photovoltaic effect in $\mathrm{LaTiO}_{3}$ films", $\mathrm{LaTiO}_{3}$ (LTO) films grown epitaxially by physical layer deposition (PLD) on $\mathrm{SrTiO}_{3}$ (STO) substrate have shown great potential application in NIR position-sensitive detector. The irradiated as-grown LTO/STO structure presented a near infrared lateral photovoltaic effect, with the open-circuit photovoltage of the LTO film dependent on the laser beam position. It was demonstrated that photosensitivity can be modified with bias current.

In "Effect of TCO/ $\mu c$-Si:H interface modification on hydrogenated microcrystalline silicon thin-film solar cells", a very thin aluminum-doped zinc oxide (AZO) coating (3-5 nm) was capped onto the textured fluorine-doped tin oxide (FTO) glass to maintain high light trapping and preserve the quality of the $\mathrm{TCO} / \mu \mathrm{c}-\mathrm{Si}: \mathrm{H}$ interface for c-Si:H single-junction solar cells. The AZO coating avoids the $\mathrm{Sn}$ reduction on the FTO surface during the Si-based thin-film deposition with hydrogen rich plasma exposure. The as-obtained $\mu \mathrm{c}-\mathrm{Si}: \mathrm{H}$ cell exhibited a significantly higher $J_{\mathrm{SC}}$ from 15.97 to $19.40 \mathrm{~mA} / \mathrm{cm}^{2}$ and an increased conversion efficiency from $5.69 \%$ to $7.09 \%$, with respect to standard FTO photoanode.

In "Effect of sulfurization temperature on properties of CZTS films by vacuum evaporation and sulfurization method," copper zinc tin sulfur thin films (CZTS) thanks to their high absorption coefficient are suitable absorbers for thin-films solar cells. CZTS were prepared on glass substrates by vacuum evaporation and sulfurization method. The optimization of the thin-films deposition and sulfurization temperature permitted a control on their crystallite size, density, and consequently the electrical properties. The authors reported $1.49 \mathrm{eV}, 9.37 \Omega \cdot \mathrm{cm}, 1.714 \times 10^{17} \mathrm{~cm}^{-3}$, and $3.89 \mathrm{~cm}^{2} /(\mathrm{Vs})$ for band gap energy, resistivity, carrier concentration, and mobility of the CZTS thin films, respectively.

In " $C-V$ calculations in $C d S / C d T e$ thin films solar cells with a $\mathrm{CdS}_{x} \mathrm{Te}_{1-x}$ interlayer", theoretical effect of interlayer ternary compound $\mathrm{CdS}_{x} \mathrm{CdTe}_{1-x}$ formation in $\mathrm{CdS} / \mathrm{CdTe}$ solar cell, due to chemical interdiffusion interface, was evaluated. The authors predicted how the ternary layer acquires the properties of CdS behaving like an N-type semiconductor. The understanding of this "additive" interlayer will be useful for the optimization and efficiency improvement of CdS/ CdTe thin-film solar cells.

Gaetano Di Marco Antonino Bartolotta Francesco Bonaccorso Stefano Caramori Giuseppe Calogero Leonardo Palmisano 

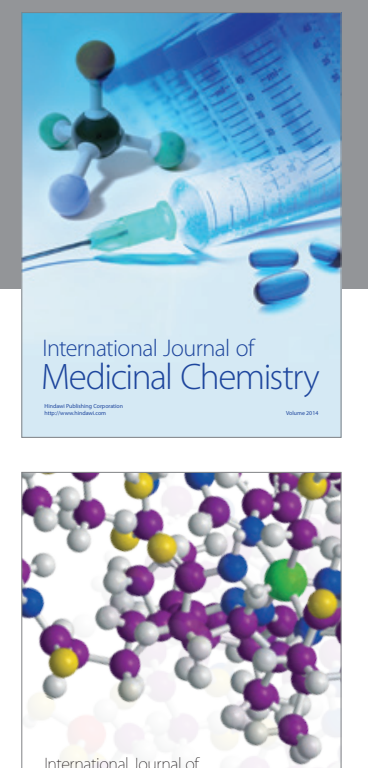

\section{Carbohydrate} Chemistry

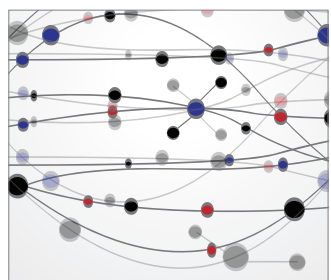

The Scientific World Journal
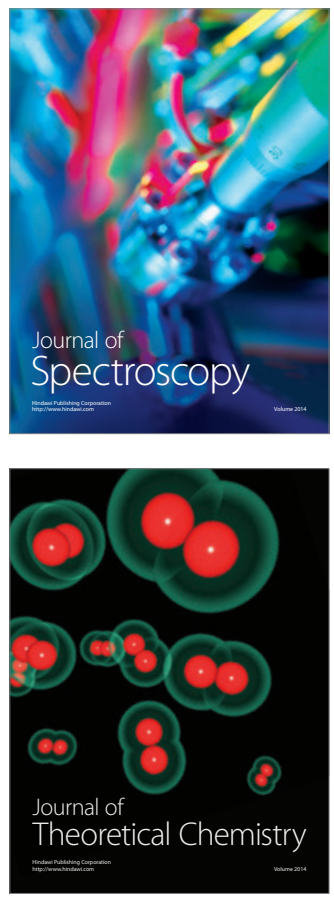
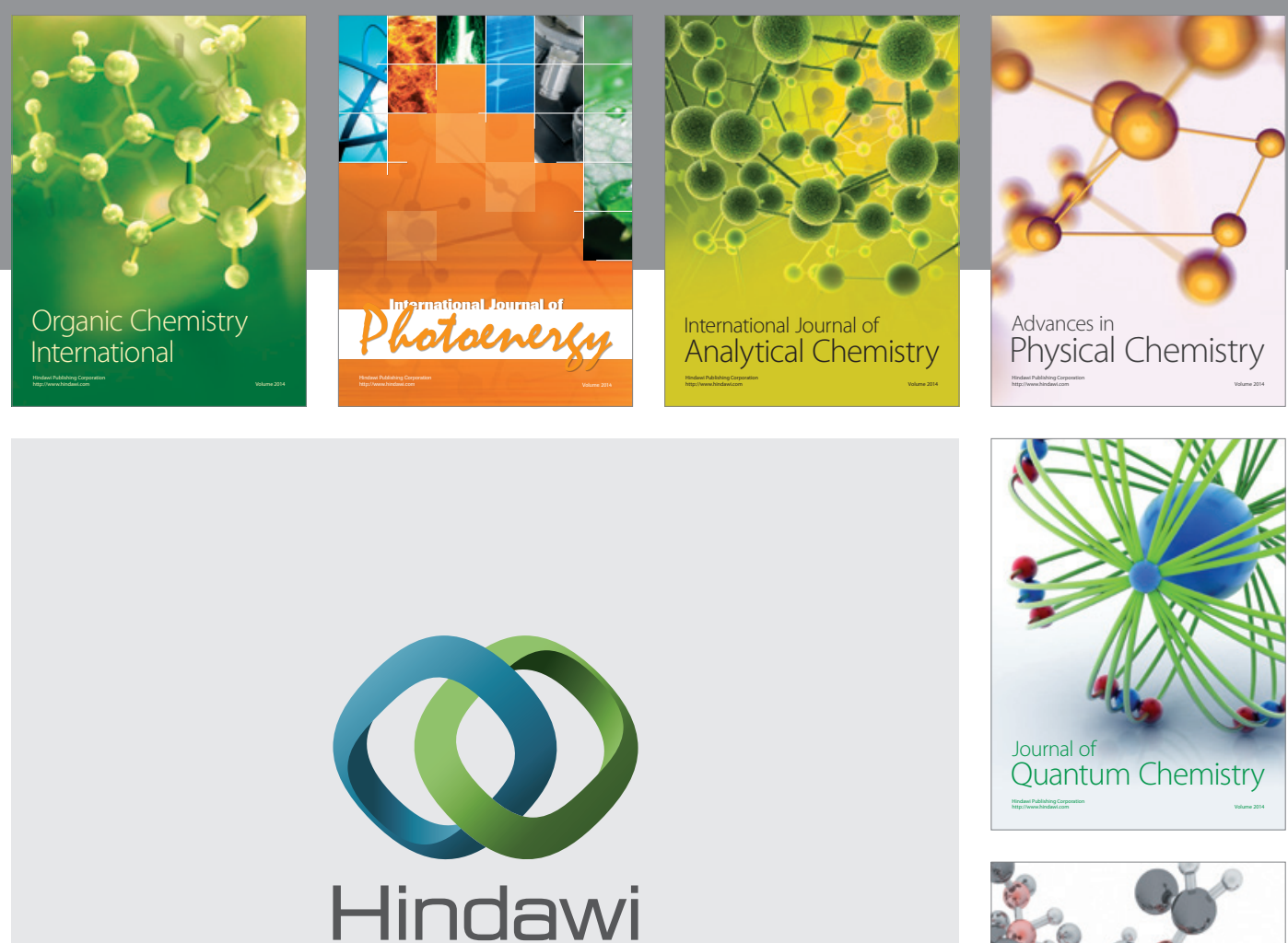

Submit your manuscripts at

http://www.hindawi.com

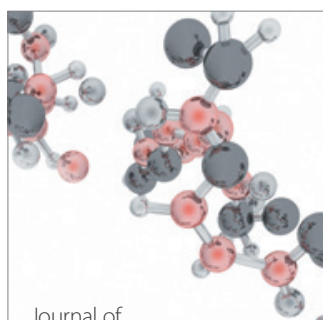

Analytical Methods

in Chemistry

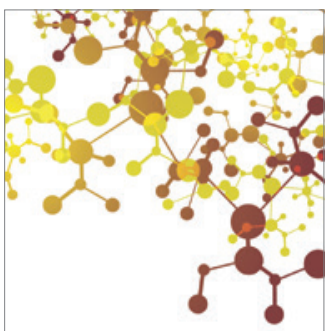

Journal of

Applied Chemistry

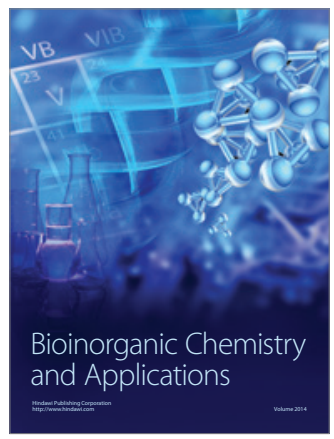

Inorganic Chemistry
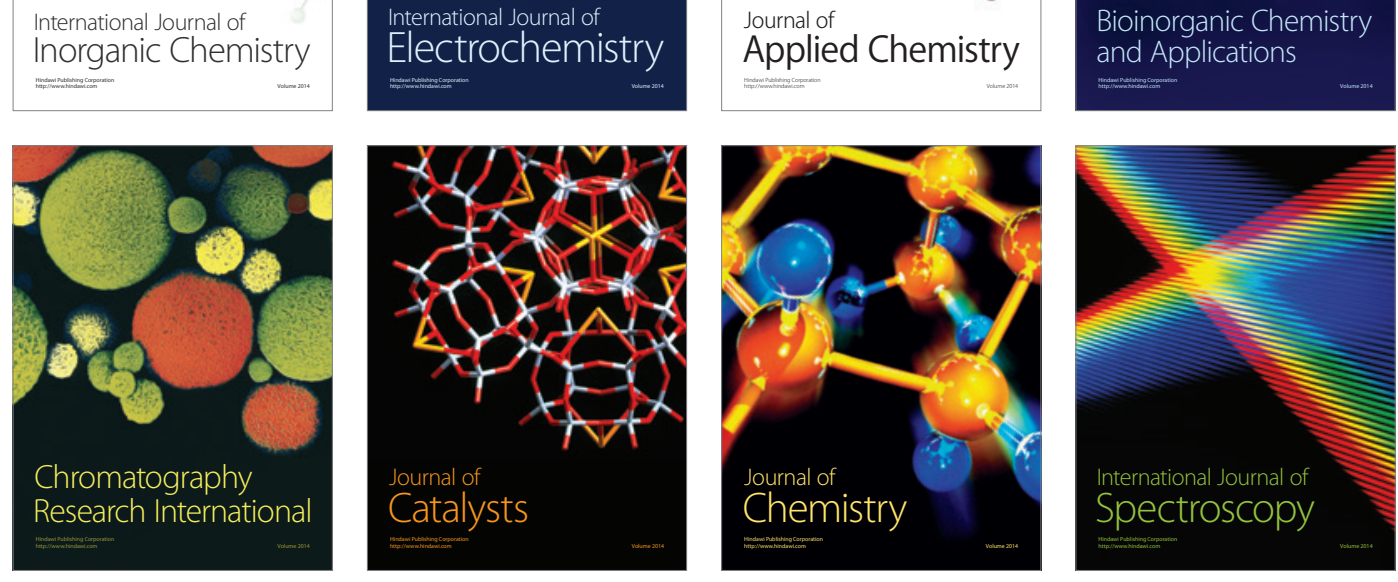